MATHEMATICS OF COMPUTATION

Volume 75 , Number 255, July 2006, Pages 1309-1318

S 0025-5718(06)01838-2

Article electronically published on March 13, 2006

\title{
TABLE OF INTEGRALS OF SQUARED JACOBIAN ELLIPTIC FUNCTIONS AND REDUCTIONS OF RELATED HYPERGEOMETRIC $R$-FUNCTIONS
}

\author{
B. C. CARLSON
}

\begin{abstract}
Any product of real powers of Jacobian elliptic functions can be written in the form $\mathrm{cs}^{m_{1}}(u, k) \mathrm{ds}^{m_{2}}(u, k) \mathrm{ns}^{m_{3}}(u, k)$. If all three $m$ 's are even integers, the indefinite integral of this product with respect to $u$ is a constant times a multivariate hypergeometric function $R_{-a}\left(b_{1}, b_{2}, b_{3} ; x, y, z\right)$ with halfodd-integral $b$ 's and $-a+b_{1}+b_{2}+b_{3}=1$, showing it to be an incomplete elliptic integral of the second kind unless all three $m$ 's are 0 . Permutations of $\mathrm{c}, \mathrm{d}$, and $\mathrm{n}$ in the integrand produce the same permutations of the variables $\{x, y, z\}=\left\{\mathrm{cs}^{2}, \mathrm{ds}^{2}, \mathrm{~ns}^{2}\right\}$, allowing as many as six integrals to take a unified form. Thirty $R$-functions of the type specified, incorporating 136 integrals, are reduced to a new choice of standard elliptic integrals obtained by permuting $x, y$, and $z$ in $R_{D}(x, y, z)=R_{-3 / 2}\left(\frac{1}{2}, \frac{1}{2}, \frac{3}{2} ; x, y, z\right)$, which is symmetric in its first two variables and has an efficient algorithm for numerical computation.
\end{abstract}

\section{INTRODUCTION}

We shall suppress the variables $(u, k)$ of the twelve Jacobian elliptic functions for brevity, and the integrals will be indefinite integrals with respect to $u$. Any one of the functions can be expressed in terms of the three that Neville [Ne, pp. 61, 178] called the primitive functions: cs, ds, and ns. For example, $\operatorname{sn}(u, k)=\mathrm{sn}=1 / \mathrm{ns}$, $\mathrm{cn}=\mathrm{cs} / \mathrm{ns}$, and $\mathrm{dn}=\mathrm{ds} / \mathrm{ns}$. Consequently, any product of real powers of Jacobian functions can be written as $\mathrm{cs}^{m_{1}} \mathrm{ds}^{m_{2}} \mathrm{~ns}^{m_{3}}$. Unless the sum of the $m$ 's is an odd positive integer, Theorem 1 will express the integral of this product as a constant times a hypergeometric $R$-function of the variables $\mathrm{cs}^{2}, \mathrm{ds}^{2}$, and $\mathrm{ns}^{2}$.

The hypergeometric $R$-function (of three variables in the present context),

$$
R_{-a}\left(b_{1}, b_{2}, b_{3} ; z_{1}, z_{2}, z_{3}\right)
$$

is defined [Ca, $\S 5.1, \S 5.2$, and (5.9-1)] as the integral average of $z^{-a}$ over the convex hull of $\left\{z_{1}, z_{2}, z_{3}\right\}$ with respect to a Dirichlet distribution with parameters $\left(b_{1}, b_{2}, b_{3}\right)$. This representation can be used if the $b$ 's and $z$ 's have positive real parts. $R_{-a}$ is homogeneous of degree $-a$ in the $z$ 's and is unchanged by simultaneous permutation of the $b$ 's and $z$ 's. More briefly, it is symmetric in the subscripts 1,2, and 3. By [Ca, (5.9-2)] it satisfies a multivariate generalization of

Received by the editor May 5, 2005.

2000 Mathematics Subject Classification. Primary 33E05, 33C75; Secondary 33C70, 33 C65.

Key words and phrases. Jacobian elliptic function, hypergeometric $R$-function, elliptic integral.

This manuscript has been authored by Iowa State University of Science and Technology under contract No. W-7405-ENG-82 with the US Department of Energy.

(C)2006 American Mathematical Society 
$d z^{-a} / d z=-a z^{-a-1}:$

$$
D R_{-a}=-a R_{-a-1}, \quad D=\sum_{i=1}^{3} \partial / \partial z_{i}
$$

It has also an important single-integral representation [Ca, (6.8-6)] for $z$ 's lying in the complex plane cut along the nonpositive real axis

$$
R_{-a}\left(b_{1}, b_{2}, b_{3} ; z_{1}, z_{2}, z_{3}\right)=\frac{1}{\mathrm{~B}\left(a, a^{\prime}\right)} \int_{0}^{\infty} t^{a^{\prime}-1} \prod_{i=1}^{3}\left(t+z_{i}\right)^{-b_{i}} d t
$$

which converges if $a$ and $a^{\prime}$ are positive, where $a+a^{\prime}=c \equiv b_{1}+b_{2}+b_{3}$. If all three $z_{i}$ take a common value $z$, the integral reduces to $z^{-a}$ times the beta function $\mathrm{B}\left(a, a^{\prime}\right)$, and $R_{-a}$ reduces to $z^{-a}$. The $R$-function is defined only if $c \neq 0,-1,-2, \ldots$ (see Ca, $\S 6.8]$ ).

The cases with $a^{\prime}=1$ (whence $-a=1-c$ ) are relatively simple in several respects. In particular the derivative is then an elementary function by (1.2) and [Ca, (6.6-5)]:

$$
D R_{1-c}=(1-c) R_{-c}=(1-c) \prod_{i=1}^{3} z_{i}^{-b_{i}} .
$$

It is (1.4) that allows the integral of $\mathrm{cs}^{m_{1}} \mathrm{ds}^{m_{2}} \mathrm{~ns}^{m_{3}}, m_{1}+m_{2}+m_{3} \neq 1,3,5, \ldots$, to be represented as a function of type $R_{1-c}$, as will be proved in Theorem 1 .

If $m_{1}, m_{2}$, and $m_{3}$ are even integers, not all 0 , we shall find that the integral of $\mathrm{cs}^{m_{1}} \mathrm{ds}^{m_{2}} \mathrm{~ns}^{m_{3}}$ is an elliptic integral of the second kind. It can be expressed in terms of standard integrals (and rational functions of cs, ds, and ns), and the conventional choice, as in [BF, 361.01-361.39], consists of

$$
u=R_{-1 / 2}\left(\frac{1}{2}, \frac{1}{2}, \frac{1}{2} ; \mathrm{cs}^{2}, \mathrm{ds}^{2}, \mathrm{~ns}^{2}\right), \quad E(u)=R_{-1 / 2}\left(\frac{1}{2},-\frac{1}{2}, \frac{3}{2} ; \mathrm{cs}^{2}, \mathrm{ds}^{2}, \mathrm{~ns}^{2}\right) .
$$

Both functions obviously have $a^{\prime}=1$ since $a^{\prime}$ is the sum of all the visible parameters. Although $u$, an integral of the first kind, has equal $b$-parameters and is therefore symmetric in c, d, and n, this symmetry is absent in $E(u)$ where the $b$ 's are all different. Therefore $E(u)$ is not suitable for obtaining reduction formulas that remain valid when c, d, and $\mathrm{n}$ are permuted. The same remark applies if $E(u)$ is replaced by another integral of the second kind with $a^{\prime}=1$ :

$$
R_{D}(u)=R_{-3 / 2}\left(\frac{1}{2}, \frac{1}{2}, \frac{3}{2} ; \mathrm{cs}^{2}, \mathrm{ds}^{2}, \mathrm{~ns}^{2}\right),
$$

which has two equal $b$ 's and so is symmetric in $\mathrm{c}$ and $\mathrm{d}$ but not in $\mathrm{n}$.

Replacement of $E(u)$ by the completely symmetric integral of the second kind,

$$
R_{G}(u)=R_{1 / 2}\left(\frac{1}{2}, \frac{1}{2}, \frac{1}{2} ; \mathrm{cs}^{2}, \mathrm{ds}^{2}, \mathrm{~ns}^{2}\right),
$$

is the choice made in a different context in [Ca, Table 9.3-1] and also in [NC, Table II], a table that reduces nine integrals of the second kind with $a^{\prime}=1$ among other cases. Unfortunately $R_{G}$, with $a^{\prime}=2$, has more complicated properties and less satisfactory methods of numerical computation than $R_{D}$. Although reductions to $u$ and $R_{G}(u)$ remain valid under permutation of $\mathrm{c}$, $\mathrm{d}$, and $\mathrm{n}$, their coefficients in the formulas tend to be rather cumbersome and are not always functions of $k$ alone.

A novel and perhaps more satisfactory choice is to replace both $u$ and $E(u)$ by three integrals of the second kind: $R_{D}(u)$ and the two functions obtained from it 
by exchanging $\mathrm{n}$ with $\mathrm{c}$ or $\mathrm{d}$. That is to say, we define

$$
R_{D}(x, y, z)=R_{-3 / 2}\left(\frac{1}{2}, \frac{1}{2}, \frac{3}{2} ; x, y, z\right),
$$

which has an efficient algorithm for numerical computation as a function of real or complex $x, y$, and $z$ (see [num, p.20]), and use as standard integrals

$$
R_{D}(y, z, x), \quad R_{D}(z, x, y), \quad R_{D}(x, y, z), \quad\{x, y, z\}=\left\{\mathrm{cs}^{2}, \mathrm{ds}^{2}, \mathrm{~ns}^{2}\right\} .
$$

This choice permits reduction formulas that unify integrals differing only by permutations of $\mathrm{c}$, d, and $\mathrm{n}$. Also, the coefficient of each $R_{D}$ depends only on $k$. For checking against other tables, it is useful to note that

$$
\begin{gathered}
\frac{k^{2}}{3} R_{D}\left(\mathrm{cs}^{2}, \mathrm{ds}^{2}, \mathrm{~ns}^{2}\right)=u-E(u) \\
\frac{k^{2} k^{\prime 2}}{3} R_{D}\left(\mathrm{~ns}^{2}, \mathrm{cs}^{2}, \mathrm{ds}^{2}\right)=-k^{\prime 2} u+E(u)-\frac{k^{2} \mathrm{cs}}{\mathrm{ds} \mathrm{ns}}, \quad k^{\prime 2}=1-k^{2} \\
\frac{k^{\prime 2}}{3} R_{D}\left(\mathrm{ds}^{2}, \mathrm{~ns}^{2}, \mathrm{cs}^{2}\right)=-E(u)+\frac{\mathrm{ds}}{\mathrm{cs} \mathrm{ns}} \\
1 / \sqrt{x y z}=R_{-3 / 2}\left(\frac{1}{2}, \frac{1}{2}, \frac{1}{2} ; x, y, z\right)=\frac{1}{3} \sum R_{D}(y, z, x) \\
u=R_{-1 / 2}\left(\frac{1}{2}, \frac{1}{2}, \frac{1}{2} ; x, y, z\right)=R_{F}(x, y, z)=\frac{1}{3} \sum x R_{D}(y, z, x), \\
R_{G}(u)=R_{1 / 2}\left(\frac{1}{2}, \frac{1}{2}, \frac{1}{2} ; x, y, z\right)=R_{G}(x, y, z)=\frac{1}{6} \sum x(y+z) R_{D}(y, z, x)
\end{gathered}
$$

where $R_{F}$ is the symmetric incomplete elliptic integral of the first kind and the summations extend over the three cyclic permutations of $x, y$, and $z$.

To prove (1.10), (1.11), and (1.12), exchange $x$ and $y$ (and $b_{1}$ and $b_{2}$ ) in the first line of [Ca, Table 9.3-1] to get $E(u)$. Then eliminate $R_{G}$ between this and each of the three forms of the third line obtained by permuting $x, y$, and $z$ (and the $b$ 's) to get the functions in (1.9). Equations (1.13) and (1.14) are the special cases $\mathbf{b}=\left(\frac{1}{2}, \frac{1}{2}, \frac{1}{2}\right)$ of (4.7) and (4.8), respectively; (1.13) shows why the choice (1.9) of standard integrals does not lead to unique reduction formulas but can be used to choose a reduction that shows any symmetry resulting from equalities between $m_{1}$, $m_{2}$, and $m_{3}$. Equation (1.15) will be proved in $\S 3.4$.

\section{TABLE OF REDUCTIONS}

Table 1 lists reductions of some hypergeometric $R$-functions with $a^{\prime}=1$ :

$$
R_{1-c}\left(b_{1}, b_{2}, b_{3} ; x, y, z\right), \quad c=\sum_{i=1}^{3} b_{i} \neq 0,-1,-2, \ldots .
$$

Because the parameters $a, b_{1}, b_{2}, b_{3}$ are half-odd-integers in each case while $a^{\prime}$ is a positive integer and (1.14) is omitted, the functions are incomplete elliptic integrals of the second kind (see [Ca, pp. 259-262]). By Theorem 1 below, this table serves to reduce many integrals of products of Jacobian elliptic functions. To unify integrals that differ only by permutations of $\mathrm{c}, \mathrm{d}$, and $\mathrm{n}$, we let

$$
\{\mathrm{p}, \mathrm{q}, \mathrm{r}\}=\{\mathrm{c}, \mathrm{d}, \mathrm{n}\}
$$

so that the table needs to include only integrands $\mathrm{ps}^{m_{1}} \mathrm{qs}^{m_{2}} \mathrm{rs}^{m_{3}}$ with $m_{1} \geq m_{2} \geq$ $m_{3}$. If two $m$ 's are equal, then $\mathrm{ps}^{2} \mathrm{qs}^{2} \mathrm{rs}^{-4}$, for example, can be made to represent $\mathrm{ds}^{2} \mathrm{~ns}^{2} \mathrm{cs}^{-4}$ or $\mathrm{ns}^{2} \mathrm{cs}^{2} \mathrm{ds}^{-4}$ or $\mathrm{cs}^{2} \mathrm{ds}^{2} \mathrm{~ns}^{-4}$ by choosing (p,q,r) to be $(\mathrm{d}, \mathrm{n}, \mathrm{c})$ or $(\mathrm{n}, \mathrm{c}, \mathrm{d})$ 
or $(\mathrm{c}, \mathrm{d}, \mathrm{n})$, respectively. If the $m$ 's are all different, six integrands can be represented by one. Although Table 1 lists only cases where the $m$ 's are even integers, we begin with a theorem that is less restrictive.

Theorem 1. Let $m_{1}, m_{2}$, and $m_{3}$ be real numbers, $M=\sum_{i=1}^{3} m_{i} \neq 1,3,5,7, \ldots$, $b_{i}=\left(1-m_{i}\right) / 2$ for $i=1,2,3$, and $c=\sum_{i=1}^{3} b_{i}$. Then

$$
\int \mathrm{ps}^{m_{1}} \mathrm{qs}^{m_{2}} \mathrm{rs}^{m_{3}} d u=\frac{1}{1-M} R_{1-c}\left(b_{1}, b_{2}, b_{3} ; \mathrm{ps}^{2}, \mathrm{qs}^{2}, \mathrm{rs}^{2}\right) .
$$

Remark. As noted earlier, the $R$-function is not defined if $c=0,-1,-2, \ldots$ Since $c=(3-M) / 2$ we require $M \neq 3,5,7, \ldots$.

Proof. Define

$$
J=R_{1-c}\left(b_{1}, b_{2}, b_{3} ; \mathrm{ps}^{2}, \mathrm{qs}^{2}, \mathrm{rs}^{2}\right) .
$$

Since $\mathrm{cs}^{2}=\mathrm{ns}^{2}-1$ and $\mathrm{ds}^{2}=\mathrm{ns}^{2}-k^{2}$, it follows that $\mathrm{ps}^{2}, \mathrm{qs}^{2}$, and $\mathrm{rs}^{2}$ differ by constants and have the same derivative [cdn, (2.3)] with respect to $u$ :

$$
\frac{d}{d u} \mathrm{ps}^{2}=\frac{d}{d u} \mathrm{qs}^{2}=\frac{d}{d u} \mathrm{rs}^{2}=-2(\mathrm{ps})(\mathrm{qs})(\mathrm{rs}) .
$$

Then (1.2) and (1.4) with $\left(z_{1}, z_{2}, z_{3}\right)=\left(\mathrm{ps}^{2}, \mathrm{qs}^{2}, \mathrm{rs}^{2}\right)$ imply

$$
\begin{aligned}
\frac{d J}{d u} & =(D J) \frac{d}{d u} \mathrm{ps}^{2} \\
& =-2(\mathrm{ps})(\mathrm{qs})(\mathrm{rs})(1-c)(\mathrm{ps})^{-2 b_{1}}(\mathrm{qs})^{-2 b_{2}}(\mathrm{rs})^{-2 b_{3}} \\
& =(2 c-2) \mathrm{ps}^{m_{1}} \mathrm{qs}^{m_{2}} \mathrm{rs}^{m_{3}} .
\end{aligned}
$$

Since $2 c-2=1-M$, integration proves (2.3).

\subsection{Summary of notation for Table 1.}

$$
M=\sum_{i=1}^{3} m_{i}, \quad c=\sum_{i=1}^{3} b_{i}, \quad m_{i}+2 b_{i}=1, \quad M+2 c=3, \quad 1-M=2(c-1) .
$$

In Table 1 the column labeled $\mathbf{m}$ shows $m_{1}, m_{2}, m_{3}$, and the column labeled $2 \mathbf{b}$ shows $2 b_{1}, 2 b_{2}, 2 b_{3}$.

$$
\begin{gathered}
(x, y, z)=\left(\mathrm{ps}^{2}, \mathrm{qs}^{2}, \mathrm{rs}^{2}\right), \quad\{\mathrm{p}, \mathrm{q}, \mathrm{r}\}=\{\mathrm{c}, \mathrm{d}, \mathrm{n}\}, \\
C_{0} R_{1-c}\left(b_{1}, b_{2}, b_{3} ; x, y, z\right) \\
=C_{x} R_{D}(y, z, x)+C_{y} R_{D}(z, x, y)+C_{z} R_{D}(x, y, z)+C_{a} / \sqrt{x y z}, \\
P=x-y=\mathrm{ps}^{2}-\mathrm{qs}^{2}=\Delta(\mathrm{p}, \mathrm{q}), \quad \Delta(\mathrm{n}, \mathrm{c})=-\Delta(\mathrm{c}, \mathrm{n})=1, \\
Q=y-z=\mathrm{qs}^{2}-\mathrm{rs}^{2}=\Delta(\mathrm{q}, \mathrm{r}), \quad \Delta(\mathrm{n}, \mathrm{d})=-\Delta(\mathrm{d}, \mathrm{n})=k^{2}, \\
R=z-x=\mathrm{rs}^{2}-\mathrm{ps}^{2}=\Delta(\mathrm{r}, \mathrm{p}), \quad \Delta(\mathrm{d}, \mathrm{c})=-\Delta(\mathrm{c}, \mathrm{d})=k^{\prime 2}=1-k^{2} . \\
P+Q+R=0, \quad z P+x Q+y R=0 .
\end{gathered}
$$


TABLE 1. Reductions of $(1-M) \int \mathrm{ps}^{m_{1}} \mathrm{qs}^{m_{2}} \mathrm{rs}^{m_{3}} d u=R_{1-c}\left(b_{1}, b_{2}, b_{3} ; x, y, z\right)$. For notation see Section 2.1.

\begin{tabular}{|c|c|c|c|c|c|c|c|c|}
\hline $\mathbf{m}$ & $1-M$ & $2 \mathbf{b}$ & $1-c$ & $C_{0}$ & $\overline{C_{x}}$ & $C_{y}$ & $C_{z}$ & $C_{a}$ \\
\hline$-2,-2,-2$ & 7 & $3,3,3$ & $-7 / 2$ & $-3 P Q R / 7$ & $Q$ & $R$ & $P$ & 0 \\
\hline $0,-2,-4$ & 7 & $1,3,5$ & $-7 / 2$ & $9 Q^{2} R / 7$ & $-Q$ & $4 R$ & $Q-4 R$ & $3 Q R / z$ \\
\hline $0,0,-6$ & 7 & $1,1,7$ & $-7 / 2$ & $45 Q^{2} R^{2} / 7$ & $Q R-4 Q^{2}$ & $Q R-4 R^{2}$ & $4 P^{2}-10 Q R$ & $3 Q R(z Q-z R+3 Q R) / z^{2}$ \\
\hline $0,-2,-2$ & 5 & $1,3,3$ & $-5 / 2$ & $3 Q / 5$ & 0 & -1 & 1 & 0 \\
\hline $0,0,-4$ & 5 & $1,1,5$ & $-5 / 2$ & $9 Q R / 5$ & $-Q$ & $R$ & $Q-R$ & $3 Q R / z$ \\
\hline $2,-2,-4$ & 5 & $-1,3,5$ & $-5 / 2$ & $9 Q^{2} / 5$ & 0 & $4 P$ & $3 R-P$ & $3 x Q / z$ \\
\hline $2,0,-6$ & 5 & $-1,1,7$ & $-5 / 2$ & $9 Q^{2} R / P$ & $Q$ & $-4 R$ & $4 R-Q$ & $3 Q R(3 x Q-z P) / z^{2} P$ \\
\hline $4,-2,-6$ & 5 & $-3,3,7$ & $-5 / 2$ & $3 Q^{3} / P$ & 0 & $R-7 P$ & $P-7 R$ & $3 x Q(-z P+x Q+z R) / z^{2} P$ \\
\hline $0,0,-2$ & 3 & $1,1,3$ & $-3 / 2$ & 1 & 0 & 0 & 1 & 0 \\
\hline $2,-2,-2$ & 3 & $-1,3,3$ & $-3 / 2$ & $Q$ & 0 & $-P$ & $-R$ & 0 \\
\hline $2,0,-4$ & 3 & $-1,1,5$ & $-3 / 2$ & $3 Q$ & 0 & $P$ & $-P$ & $3 x Q / z$ \\
\hline $2,2,-6$ & 3 & $-1,-1,7$ & $-3 / 2$ & $15 Q R / P$ & $Q$ & $R$ & $P$ & $3 Q R(5 x y-x Q+y R) / z^{2} P$ \\
\hline $4,0,-6$ & 3 & $-3,1,7$ & $-3 / 2$ & $15 Q^{2} / P$ & 0 & $2 Q-4 P$ & $Q+4 P$ & $3 x Q(5 x Q-z P+2 Q R) / z^{2} P$ \\
\hline $4,-2,-4$ & 3 & $-3,3,5$ & $-3 / 2$ & $3 Q^{2} / P$ & 0 & $3 P-R$ & $4 R$ & $-3 x Q R / z P$ \\
\hline $2,0,-2$ & 1 & $-1,1,3$ & $-1 / 2$ & 3 & 0 & $-P$ & 0 & $3 x$ \\
\hline $2,2,-4$ & 1 & $-1,-1,5$ & $-1 / 2$ & 9 & $P$ & $-P$ & 0 & $3(3 x y-x Q+y R) / z$ \\
\hline $4,-2,-2$ & 1 & $-3,3,3$ & $-1 / 2$ & $3 Q$ & 0 & $P R$ & $-P R$ & $3 x Q$ \\
\hline $4,0,-4$ & 1 & $-3,1,5$ & $-1 / 2$ & $9 Q / P$ & 0 & $P-2 Q$ & $R$ & $3 x Q(3 z-R) / z P$ \\
\hline $4,2,-6$ & 1 & $-3,-1,7$ & $-1 / 2$ & $45 Q / P$ & $4 Q$ & $P-4 Q$ & $-P$ & $3 Q\left(15 x y z-5 x z Q-z P R+3 y R^{2}\right) / z^{2} P$ \\
\hline $6,0,-6$ & 1 & $-5,1,7$ & $-1 / 2$ & $45 Q^{2} / P$ & 0 & $3 P Q-4 P^{2}-8 Q^{2}$ & $4 P^{2}-4 Q^{2}$ & $3 x Q\left(Q\left(15 z^{2}-10 x R-7 R^{2}\right)+z P R\right) / z^{2} P$ \\
\hline $2,0,0$ & -1 & $-1,1,1$ & $1 / 2$ & 3 & $P R$ & 0 & 0 & $3 y z$ \\
\hline $2,2,-2$ & -1 & $-1,-1,3$ & $1 / 2$ & 3 & $P R$ & $P Q$ & 0 & $3\left(z^{2}+Q R\right)$ \\
\hline $4,0,-2$ & -1 & $-3,1,3$ & $1 / 2$ & 3 & $P R$ & $-P R$ & 0 & $3(y z+x R)$ \\
\hline $4,2,-4$ & -1 & $-3,-1,5$ & $1 / 2$ & $9 / P$ & $4 R$ & $3 Q-R$ & 0 & $3\left(3 z^{3}+3 x z R-z P R+4 z Q R-Q R^{2}\right) / z P$ \\
\hline $6,-2,-2$ & -1 & $-5,3,3$ & $1 / 2$ & $3 Q / P R$ & $Q$ & $R$ & $P$ & $3 Q(y z-x P+x R) / P R$ \\
\hline $6,0,-4$ & -1 & $-5,1,5$ & $1 / 2$ & $9 Q / P R$ & $4 Q$ & $P-4 Q$ & $-P$ & $3 Q\left(4 y z^{2}-x^{3}-x z Q+3 x z R\right) / z P R$ \\
\hline $2,2,0$ & -3 & $-1,-1,1$ & $3 / 2$ & $3 / Q R$ & $P$ & $-P$ & 0 & $3 z(x y+y Q-x R) / Q R$ \\
\hline $4,0,0$ & -3 & $-3,1,1$ & $3 / 2$ & $3 / P R$ & 0 & $2 R-P$ & $R-2 P$ & $3 x\left(x^{2}+y P-z R-(P-R)^{2}\right) / P R$ \\
\hline $4,2,-2$ & -3 & $-3,-1,3$ & $3 / 2$ & $3 / P R$ & $Q-3 R$ & $-4 Q$ & 0 & $3\left(x^{2} y-y z P-4 z^{2} R-4 Q R^{2}\right) / P R$ \\
\hline $6,0,-2$ & -3 & $-5,1,3$ & $3 / 2$ & $3 / P R$ & $2 P-4 R$ & $P+4 R$ & 0 & $3\left(x^{2} y+2 y z P-4 z^{2} R+3 x P R-4 Q R^{2}\right) / P R$ \\
\hline
\end{tabular}


Without reference to Jacobian elliptic functions, Table 1 also provides a table of reductions of $R$-functions with $a^{\prime}=1$ and variables $x, y, z$. Functions with $a^{\prime}=2,3,4, \ldots$ can be obtained by successive applications of [Ca, (5.9-7)] (see $\left.\S 3.4\right)$. Functions with $a^{\prime}=0,-1,-2, \ldots$ are elementary and can be obtained directly from Ca, (6.8-15) and (6.2-1)].

\section{EXAmPles}

3.1. Example: Integral of any single squared Jacobian function. For $\left(m_{1}\right.$, $\left.m_{2}, m_{3}\right)=(0,0,-2),(2,0,-2)$, and $(2,0,0)$, Table 1 gives, respectively,

$$
\begin{aligned}
& \int \mathrm{sr}^{2} d u=\frac{1}{3} R_{D}\left(\mathrm{ps}^{2}, \mathrm{qs}^{2}, \mathrm{rs}^{2}\right), \\
& \int \mathrm{pr}^{2} d u=\frac{1}{3} \Delta(\mathrm{q}, \mathrm{p}) R_{D}\left(\mathrm{rs}^{2}, \mathrm{ps}^{2}, \mathrm{qs}^{2}\right)+\frac{\mathrm{ps}}{\mathrm{qs} \mathrm{rs}}, \\
& \int \mathrm{ps}^{2} d u=\frac{1}{3} \Delta(\mathrm{p}, \mathrm{q}) \Delta(\mathrm{p}, \mathrm{r}) R_{D}\left(\mathrm{qs}^{2}, \mathrm{rs}^{2}, \mathrm{ps}^{2}\right)-\frac{\mathrm{qs} \mathrm{rs}}{\mathrm{ps}},
\end{aligned}
$$

where $\{\mathrm{p}, \mathrm{q}, \mathrm{r}\}=\{\mathrm{c}, \mathrm{d}, \mathrm{n}\}$, the $\Delta$ 's are functions of $k^{2}$ given by $(2.9)-(2.11)$, and $R_{D}$ is symmetric in its first two variables. We illustrate the derivation of these equations by considering $\left(m_{1}, m_{2}, m_{3}\right)=(2,0,0)$. Table 1 gives

$$
\begin{gathered}
(-1) \int \mathrm{ps}^{2} \mathrm{qs}^{0} \mathrm{rs}^{0} d u=\frac{1}{3}\left[\operatorname{PR} R_{D}(y, z, x)+3 y z / \sqrt{x y z}\right], \\
\int \mathrm{ps}^{2} d u=-\frac{1}{3}\left[\Delta(p, q) \Delta(\mathrm{r}, p) R_{D}\left(\mathrm{qs}^{2}, \mathrm{rs}^{2}, \mathrm{ps}^{2}\right)+3 \mathrm{qs} \mathrm{rs} / \mathrm{ps}\right]
\end{gathered}
$$

in agreement with (3.3).

The three cases of (3.1), for $\mathrm{r}=\mathrm{n}$, $\mathrm{d}$, or $\mathrm{c}$, are simple and similar to one another, while the corresponding reductions to $E(u)$ and $u$, which follow by inspection from (1.10)-(1.12), are quite dissimilar. They are given in BF, (310.02), (318.02), and (316.02)]; note that $[\mathrm{BF}$ uses th in place of sc. Likewise (3.3), with $\mathrm{p}=\mathrm{n}$, $\mathrm{d}$, or $\mathrm{c}$ and with $\Delta$ 's taken from (2.9)-(2.11), agrees with and can replace $\mathrm{BF}$, (311.02), (319.02), and (317.02)]. Finally (3.2), with pr $=\mathrm{cn}, \mathrm{nc}$, dn, nd, cd, or dc, agrees with and can replace six integrals in $\mathrm{BF}, 3 \mathrm{XX} .02, \mathrm{XX}=12,13,14,15,20$, and 21].

3.2. Example: Integral of a product of squared Jacobian functions. Since $\mathrm{sr}^{2} \mathrm{pr}^{2}=\mathrm{ps}^{2} \mathrm{qs}^{0} \mathrm{rs}^{-4}$, Table 1 gives

$$
\begin{aligned}
3 \int \mathrm{sr}^{2} \mathrm{pr}^{2} d u & =R_{-3 / 2}\left(-\frac{1}{2}, \frac{1}{2}, \frac{5}{2} ; \mathrm{ps}^{2}, \mathrm{qs}^{2}, \mathrm{rs}^{2}\right) \\
=\frac{1}{3 Q}\left[P R_{D}\left(\mathrm{rs}^{2}, \mathrm{ps}^{2}, \mathrm{qs}^{2}\right)-P R_{D}\left(\mathrm{ps}^{2}, \mathrm{qs}^{2}, \mathrm{rs}^{2}\right)+\frac{3 x Q}{z \sqrt{x y z}}\right] & \\
\int \mathrm{sr}^{2} \mathrm{pr}^{2} d u & =\frac{\Delta(\mathrm{p}, \mathrm{q})}{9 \Delta(\mathrm{q}, \mathrm{r})}\left[R_{D}\left(\mathrm{rs}^{2}, \mathrm{ps}^{2}, \mathrm{qs}^{2}\right)-R_{D}\left(\mathrm{ps}^{2}, \mathrm{qs}^{2}, \mathrm{rs}^{2}\right)\right]+\frac{\mathrm{ps}}{3 \mathrm{qs}^{3}}
\end{aligned}
$$

This formula can replace [BF, 361.01, .02, .07, .15, .19, and .27]. For instance, if $(\mathrm{p}, \mathrm{q}, \mathrm{r})=(\mathrm{c}, \mathrm{d}, \mathrm{n})$ it becomes

$$
\int \mathrm{sn}^{2} \mathrm{cn}^{2} d u=\frac{k^{\prime 2}}{9 k^{2}}\left[R_{D}\left(\mathrm{~ns}^{2}, \mathrm{cs}^{2}, \mathrm{ds}^{2}\right)-R_{D}\left(\mathrm{cs}^{2}, \mathrm{ds}^{2}, \mathrm{~ns}^{2}\right)\right]+\frac{\mathrm{cs}}{3 \mathrm{ds} \mathrm{ns}^{3}},
$$

which agrees with $[\mathrm{BF}, 361.01]$ after substitution of (1.11) and (1.10). 
3.3. Example: Reducing an $R$-function with $a^{\prime}=1$. If the objective is to reduce an $R$-function with $a^{\prime}=1$, the variables $x, y, z$ should be used throughout. For instance, the row of Table 1 with $\left(2 b_{1}, 2 b_{2}, 2 b_{3}\right)=(-1,1,1)$ gives

$$
3 R_{1 / 2}\left(-\frac{1}{2}, \frac{1}{2}, \frac{1}{2} ; x, y, z\right)=(x-y)(z-x) R_{D}(y, z, x)+3 y z / \sqrt{x y z} .
$$

This reduction is used in the next example. Because $b_{2}=b_{3}$ we expect symmetry of (3.5) in $y$ and $z$, which is indeed evident.

3.4. Example: Reducing an $R$-function with $a^{\prime}>1$. As mentioned in the last sentence of Section 2.1, functions with $a^{\prime}=2,3,4, \ldots$ can be obtained by successive applications of [Ca, (5.9-7)] with parameters $t=-a, \mathbf{b}=\left(b_{1}, b_{2}, b_{3}\right)$, and $\mathbf{z}=(x, y, z)=\left(z_{1}, z_{2}, z_{3}\right) ; \mathbf{e}_{i}$ is a triple with 1 in the $i$ th place and 0 's elsewhere. Replacing $\mathbf{b}$ by $\mathbf{b}-\mathbf{e}_{i}$ (and $c$ by $c-1$ ), we have

$$
\left(a^{\prime}-1\right) R_{-a}(\mathbf{b}, \mathbf{z})=(c-1) R_{-a}\left(\mathbf{b}-\mathbf{e}_{i}, \mathbf{z}\right)-a z_{i} R_{-a-1}(\mathbf{b}, \mathbf{z}) .
$$

For instance, choosing $a^{\prime}=2, \mathbf{b}=\left(\frac{1}{2}, \frac{1}{2}, \frac{1}{2}\right)$ and $i=1$, we find $a=-\frac{1}{2}$ and

$$
R_{1 / 2}\left(\frac{1}{2}, \frac{1}{2}, \frac{1}{2} ; x, y, z\right)=\frac{1}{2} R_{1 / 2}\left(-\frac{1}{2}, \frac{1}{2}, \frac{1}{2} ; x, y, z\right)+\frac{1}{2} x R_{-1 / 2}\left(\frac{1}{2}, \frac{1}{2}, \frac{1}{2} ; x, y, z\right) .
$$

The left side is $R_{G}(x, y, z)$ by (1.15). On the right side the first term is reduced by (3.5) and the second is a multiple of $R_{F}$ :

$$
6 R_{G}(x, y, z)=(x-y)(z-x) R_{D}(y, z, x)+3 y z / \sqrt{x y z}+3 x R_{F}(x, y, z) .
$$

To make the right side look symmetric in $x, y, z$, like the left side, we substitute (1.13) and (1.14) and collect terms to get

$$
\begin{aligned}
6 R_{G}(x, y, z)= & {\left[(x-y)(z-x)+y z+x^{2}\right] R_{D}(y, z, x) } \\
& +(y z+x y) R_{D}(z, x, y)+(y z+x z) R_{D}(x, y, z) .
\end{aligned}
$$

The expression in square brackets reduces to $x y+x z$, proving (1.15).

\section{Method of making Table 1}

The entries in Table 1 were found and checked by using relations between associated $R$-functions (those whose corresponding parameters differ by integers). Relations useful for present purposes involve only $R$-functions with $a^{\prime}=1$ or 0 , where $a^{\prime}$ is the sum of the exhibited parameters. If $a^{\prime}=0$ the function is algebraic in its variables by $[\mathrm{Ca},(6.6-5)]$ because the $b$-parameters are half-odd integers. If $a^{\prime}=1$, the representation by a Dirichlet average [Ca, (5.9-1)] would be excluded by a negative $b$-parameter and the single-integral representation (1.3) would be excluded if $1-c>0$. Relations that are useful in making or extending Table 1 are given in the following lemma.

Lemma 1. Let $\mathbf{b}=\left(b_{1}, b_{2}, b_{3}\right), c=\sum_{i=1}^{3} b_{i}$, and $\mathbf{z}=\left(z_{1}, z_{2}, z_{3}\right)$. Let $\{i, j, k\}=$ $\{1,2,3\}$ and let $\mathbf{e}_{i}$ be a triple with 1 in the ith place and 0 's in the $j$ th and $k$ th places. (For example, $\mathbf{b}-\mathbf{e}_{2}=\left(b_{1}, b_{2}-1, b_{3}\right)$.) Then the $R$-function of three variables satisfies

$$
\begin{aligned}
& \left(1-b_{k}\right) R_{1-c}(\mathbf{b}, \mathbf{z}) \\
& \quad=b_{i} R_{1-c}\left(\mathbf{b}+\mathbf{e}_{i}-\mathbf{e}_{k}, \mathbf{z}\right)+b_{j} R_{1-c}\left(\mathbf{b}+\mathbf{e}_{j}-\mathbf{e}_{k}, \mathbf{z}\right)+(1-c) z_{k} \prod_{s=1}^{3} z_{s}^{-b_{s}},
\end{aligned}
$$




$$
\begin{aligned}
& c R_{1-c}(\mathbf{b}, \mathbf{z}) \\
& =b_{j}\left(z_{j}-z_{i}\right) R_{-c}\left(\mathbf{b}+\mathbf{e}_{j}, \mathbf{z}\right)+b_{k}\left(z_{k}-z_{i}\right) R_{-c}\left(\mathbf{b}+\mathbf{e}_{k}, \mathbf{z}\right)+c z_{i} \prod_{s=1}^{3} z_{s}^{-b_{s}}, \\
& \quad(c-2)\left(z_{i}-z_{j}\right) R_{1-c}(\mathbf{b}, \mathbf{z})=(c-1)\left[R_{2-c}\left(\mathbf{b}-\mathbf{e}_{i}, \mathbf{z}\right)-R_{2-c}\left(\mathbf{b}-\mathbf{e}_{j}, \mathbf{z}\right)\right] \\
& c R_{1-c}(\mathbf{b}, \mathbf{z}) \\
& =b_{j}\left(z_{j}-z_{i}\right) R_{-c}\left(\mathbf{b}+\mathbf{e}_{i}+\mathbf{e}_{j}-\mathbf{e}_{k}, \mathbf{z}\right) \\
& \quad-\left(b_{i}+b_{j}\right)\left(z_{k}-z_{i}\right) R_{-c}\left(\mathbf{b}+\mathbf{e}_{i}, \mathbf{z}\right)+c z_{k} \prod_{s=1}^{3} z_{s}^{-b_{s}}, \\
& \left(b_{k}-1\right) c R_{1-c}(\mathbf{b}, \mathbf{z}) \\
& =(c-1) b_{j}\left(z_{j}-z_{i}\right) R_{-c}\left(\mathbf{b}+\mathbf{e}_{i}+\mathbf{e}_{j}-\mathbf{e}_{k}, \mathbf{z}\right) \\
& -c\left(b_{i}+b_{j}\right) R_{1-c}\left(\mathbf{b}+\mathbf{e}_{i}-\mathbf{e}_{k}, \mathbf{z}\right)+c(c-1) z_{k} \prod_{s=1}^{3} z_{s}^{-b_{s}} .
\end{aligned}
$$

If $b_{i}+b_{j}=0$, then $c=b_{k}$ and

$$
b_{k} R_{1-b_{k}}(\mathbf{b}, \mathbf{z})=b_{j}\left(z_{j}-z_{i}\right) R_{-b_{k}}\left(\mathbf{b}+\mathbf{e}_{i}+\mathbf{e}_{j}-\mathbf{e}_{k}, \mathbf{z}\right)+b_{k}\left(z_{j} / z_{i}\right)^{b_{i}} z_{k}^{1-b_{k}} .
$$

Proof. Choose $t=-c$ in [Ca, (5.9-5)] and use [Ca, (6.6-5)] to get

$$
c \prod_{s=1}^{3} z_{s}^{-b_{s}}=\sum_{s=1}^{3} b_{s} R_{-c}\left(\mathbf{b}+\mathbf{e}_{s}, \mathbf{z}\right) .
$$

Then replace $\mathbf{b}$ by $\mathbf{b}-\mathbf{e}_{k}$ (and hence $c$ by $c-1$ and $b_{k}$ by $b_{k}-1$ ) to prove (4.1).

To prove (4.2) multiply both sides of (4.7) by $z_{i}$ and subtract it from the case $t=-c$ of $[\mathrm{Ca},(5.9-6)]$ :

$$
c R_{1-c}(\mathbf{b}, \mathbf{z})=\sum_{s=1}^{3} b_{s} z_{s} R_{-c}\left(\mathbf{b}+\mathbf{e}_{s}, \mathbf{z}\right) .
$$

Equation (4.3) is the first equation in [Ca, Ex. 5.9-6 and p. 305] with $t=2-c$.

To prove (4.4) and (4.5), first replace $\mathbf{b}$ by $\mathbf{b}+\mathbf{e}_{i}-\mathbf{e}_{k}$ (and hence $b_{i}$ by $b_{i}+1$ and $b_{k}$ by $\left.b_{k}-1\right)$ in (4.2) to get

$$
\begin{aligned}
& c R_{1-c}\left(\mathbf{b}+\mathbf{e}_{i}-\mathbf{e}_{k}, \mathbf{z}\right) \\
& =b_{j}\left(z_{j}-z_{i}\right) R_{-c}\left(\mathbf{b}+\mathbf{e}_{i}+\mathbf{e}_{j}-\mathbf{e}_{k}, \mathbf{z}\right) \\
& \quad+\left(b_{k}-1\right)\left(z_{k}-z_{i}\right) R_{-c}\left(\mathbf{b}+\mathbf{e}_{i}, \mathbf{z}\right)+c z_{k} \prod_{s=1}^{3} z_{s}^{-b_{s} .}
\end{aligned}
$$

Next, exchange $j$ and $k$ in (4.3) and then replace $\mathbf{b}$ by $\mathbf{b}+\mathbf{e}_{i}$ (and hence $c$ by $c+1$ ) in $(4.3)$ to get

$$
(c-1)\left(z_{i}-z_{k}\right) R_{-c}\left(\mathbf{b}+\mathbf{e}_{i}, \mathbf{z}\right)=c\left[R_{1-c}(\mathbf{b}, \mathbf{z})-R_{1-c}\left(\mathbf{b}+\mathbf{e}_{i}-\mathbf{e}_{k}, \mathbf{z}\right)\right] .
$$

Elimination of $R_{1-c}\left(\mathbf{b}+\mathbf{e}_{i}-\mathbf{e}_{k}, \mathbf{z}\right)$ or of $R_{-c}\left(\mathbf{b}+\mathbf{e}_{i}, \mathbf{z}\right)$ between (4.9) and (4.10) produces (4.4) or (4.5). Both equations have (4.6) as a special case. 
Remark. A section of the table that contains $R$-functions with the same value of $c$ will be called a level of the table. One level is higher than another if its value of $c$ is the larger of the two. For example the highest level shown consists of the first three rows of the table, in which $c=9 / 2$. Then (4.1) connects three functions in the same level, and (4.3) expresses a function in terms of two functions in the next lower level. If two $b$-parameters have sum $0,(4.6)$ expresses the function in terms of a single function in the next higher level, but otherwise (4.2) and (4.4) express it in terms of two functions in the next higher level. Finally, (4.5) expresses it in terms of another function in the same level and one in the next higher level. These observations are helpful to keep in mind while making, checking, or extending the table.

When working with specific numerical values of the parameters, it is convenient to define

$$
\left[2 b_{1}, 2 b_{2}, 2 b_{3}\right]=R_{1-c}(\mathbf{b}, \mathbf{z}) .
$$

For example $[1,1,3]=R_{-3 / 2}\left(\frac{1}{2}, \frac{1}{2}, \frac{3}{2} ; x, y, z\right)$. Since this function is $R_{D}(x, y, z)$, one of the three standard functions used in Table 1, it is a natural starting point in making the table, where it occupies the first line in the third level (with $1-c=$ $-3 / 2)$. We shall illustrate the procedure and the use of (4.1)-(4.6). To get the first line in the next higher (second) level, we use (4.3) with $(i, j, k)=(2,3,1)$ to find $\frac{3}{2}(y-z)[1,3,3]=\frac{5}{2}([1,1,3]-[1,3,1])$. By the invariance of the $R$-function under simultaneous permutations of the $b$-parameters and the variables, $[1,3,1]=$ $R_{D}(z, x, y)$, and hence

$$
(3 Q / 5)[1,3,3]=R_{D}(x, y, z)-R_{D}(x, z, y) .
$$

From this result we can get the third line in the starting (third) level by using (4.6) with $(i, j, k)=(1,2,3)$ to find $\frac{5}{2}[-1,1,5]=\frac{1}{2}(y-x)[1,3,3]+\frac{5}{2} \sqrt{x / y} z^{-3 / 2}$. Multiplication by $6 Q / 5$ gives

$$
3 Q[-1,1,5]=P R_{D}(x, z, y)-P R_{D}(x, y, z)+3 Q \sqrt{x / y} z^{-3 / 2} .
$$

An alternative path from the starting point uses (4.6) to express $[-1,1,3]$ on the next lower (fourth) level in terms of $[1,3,1]=R_{D}(z, x, y)$ and then uses (4.3) to return to the third level by expressing $[-1,3,3]$ in terms of $[-1,1,3]$ and $[-1,3,1]$, which are related by permutation.

We now outline one way of obtaining the remaining three lines shown in the third level.

- Function $[1,1,5]$ (second level) follows from $[-1,1,5]$ and $[1,1,3]$ by $(4.3)$ with $(i, j, k)=(1,3,2)$.

- Function $[-1,-1,7]$ (third level) follows from $[1,1,5]$ and $[-1,1,5]$ by $(4.5)$ with $(i, j, k)=(2,1,3)$.

- Function $[-1,3,5]$ (second level) follows from $[-1,1,5]$ and $[-1,3,3]$ by (4.3) with $(i, j, k)=(2,3,1)$.

- Function $[-3,3,5]$ (third level) follows from $[-1,3,5]$ and $[-1,5,3]$ (differing only by permutation) by (4.4) with $(i, j, k)=(1,2,3)$.

- Function $[-3,1,7]$ (third level) follows from $[-3,3,5]$ and $[-1,1,5]$ by $(4.1)$ with $(i, j, k)=(2,1,3)$.

As more lines in the table are established, more ways of checking for errors become available. For example, $[-1,1,3]$ follows alternatively from $[1,1,3]$ and $[-1,3,3]$ by $(4.2)$ with $(i, j, k)=(1,2,3)$, and also from $[1,1,3]$ and $[-1,1,5]$ by $(4.2)$ with 
$(i, j, k)=(1,3,2)$. For another example, $[-1,3,3]$ follows alternatively from $[1,1,5]$ and $[1,3,3]$ by $(4.4)$ with $(i, j, k)=(1,3,2)$. The result can then be simplified by using (1.13) to eliminate $R_{D}(y, z, x)$ and show the expected symmetry in $y$ and $z$.

For integrals of Jacobian functions the symbols $P, Q$, and $R$ should be used as much as feasible in the coefficient $C_{a}$ of $1 / \sqrt{x y z}$ because they are simple functions of the modulus. For other purposes it is straightforward to rewrite $C_{a}$ in terms of $x, y$, and $z$ by using $(2.9)-(2.11)$.

\section{REFERENCES}

[BF] P. F. Byrd and M. D. Friedman, Handbook of Elliptic Integrals for Engineers and Scientists, 2nd ed., Springer-Verlag, New York,1971. MR0277773 (43:3506)

[Ca] B. C. Carlson, Special Functions of Applied Mathematics, Academic Press, 1977. MR0590943 (58:28707)

[num] B. C. Carlson, Numerical computation of real or complex elliptic integrals, Numer. Algorithms 10(1995)13-26. MR 1345407 (97h:33035)

[cdn] B. C. Carlson, Symmetry in c, d, n of Jacobian elliptic functions, J. Math. Anal. Appl. 299(2004)242-253. MR 2091285 (2005h:33044)

[NC] W. J. Nellis and B. C. Carlson. Reduction and evaluation of elliptic integrals, Math. Comp. 20(1966)223-231. MR0215497(35:6337)

[Ne] E. H. Neville, Jacobian Elliptic Functions, 2nd ed., Oxford Univ. Press, London, 1951. MR0041934 (13:24e)

Ames Laboratory and Department of Mathematics, Iowa State University, Ames, IOWA 50011-3020

E-mail address: bcarlson@scl.ameslab.gov 